\title{
COHOMOLOGY OF GROUPS WITH METACYCLIC SYLOW $p$-SUBGROUPS
}

\author{
JILL DIETZ, JOHN MARTINO, AND STEWART PRIDDY \\ (Communicated by Thomas Goodwillie)
}

\begin{abstract}
We determine the cohomology algebras $H^{*}\left(G ; \mathbf{F}_{p}\right)$ for all groups $G$ with a metacyclic Sylow $p$-subgroup. The complete $p$-local stable decomposition of the classifying space $B G$ is also determined.
\end{abstract}

\section{INTRODUCTION AND STATEMENT OF RESULTS}

Let $P$ be a non-abelian metacyclic $p$-group of odd order and $G$ a finite group with $P$ as a Sylow $p$-subgroup. In this note we classify all possible mod- $p$ cohomology algebras $H^{*}(G)$ and determine complete $p$-local stable splittings for the classifying spaces $B G$. Much of the topological part of this work was done by the first author in $[\mathrm{D}]$; recent results on Swan groups [MP] have enabled us to show that in all cases $H^{*}(G)$ is given by a ring of invariants. Similar but less complete information for metacyclic 2-groups was obtained in [D1, MP2, M].

A metacyclic $p$-group is a $p$-group $P$ which is an extension of a cyclic group by a cyclic group. Following [D] we say that $P$ is split if $P$ can be expressed by some split extension. We recall that up to isomorphism any non-abelian metacyclic $p$-group can be expressed as

$$
P=P\left(p^{m}, p^{n}, p^{l}+1, p^{q}\right)=\left\langle x, y \mid x^{p^{m}}=1, y^{p^{n}}=x^{p^{q}}, y x y^{-1}=x^{p^{l}+1}\right\rangle
$$

for positive integers $m, n, l, q$ satisfying $l, q \leq m,\left(p^{l}+1\right)^{p^{n}} \equiv 1 \bmod p^{m},\left(p^{l}+1\right) p^{q} \equiv$ $p^{q} \bmod p^{m}, n+l \geq m$ and $q+l \geq m$. In these terms $P$ splits unless $m \neq q$ and $l<q<n[\mathrm{D}$, Thm. 3.1].

Let $W_{G}(P)=N_{G}(P) / P \cdot C_{G}(P)$; then $W_{G}(P) \leq \operatorname{Out}(P)$. If $P$ is split, then $\operatorname{Out}(P) \cong O_{p} O u t(P) \rtimes \mathbf{Z} /(p-1)$ where $O_{p} O u t(P)$ is a Sylow $p$-subgroup [D, Prop. 3.2]. Therefore $W_{G}(P)=Z / d$ where $d$ is a divisor of $p-1$. If $P$ is non-split, Out $(P)$ is a $p$-group and so $W_{G}(P)=1$. We denote by $\mathbf{F}_{p}[\cdot]$ and $E[\cdot]$ the polynomial and exterior algebras over $\mathbf{F}_{p}$.

Theorem 1.1. As an algebra, $H^{*}(G)$ has one of the following forms:

(1) If $P$ is split and $l \neq m-n$, then

$$
H^{*}(G) \cong H^{*}(P)^{W_{G}(P)}=\mathbf{F}_{p}\left[u_{d}, v\right] \otimes E\left[a_{d}, b\right]
$$

Received by the editors January 26, 1995.

1991 Mathematics Subject Classification. Primary 55R35; Secondary 20J06.

The third author is partially supported by NSF Grant DMS-9400235. 
where $\left|u_{d}\right|=2 d,|v|=2,\left|a_{d}\right|=2 d-1,|b|=1$.

(2) If $P$ is split and $l=m-n$, then

$$
H^{*}(G) \cong H^{*}(P)^{W_{G}(P)}=\mathbf{F}_{p}[v, z] \otimes E\left[b, \alpha_{2 i-1}, i=1, \ldots, p\right] / R
$$

where the relations $R$ are given by

$$
\begin{gathered}
\alpha_{2 i-1} \alpha_{2 j-1}=0, \quad 1 \leq i, j \leq p, \\
\alpha_{2 i-1} v=0, \quad 1 \leq i \leq p-1,
\end{gathered}
$$

and $|b|=1,|v|=2,|z|=2 p,\left|\alpha_{2 i-1}\right|=2 i-1+2 p d(i)$, where $0 \leq d(i)<d$ is the residue of $-i \bmod d$.

(3) If $P$ is non-split, then $H^{*}(G)=H^{*}(P)$ is isomorphic to the algebra of (1) with $d=1$ if $m=l+q$ and to that of (2) with $d=1$ if $m<l+q$.

Generators for these cohomology groups are specified explicitly in the proof.

Remark 1.2. Groups exemplifying the cases above are easily given by $G=P \rtimes \mathbf{Z} / d$.

R. Lyons has suggested other, more natural examples, which occur as automorphism groups of Chevalley groups. For example, let $\mathbf{F}_{q}$ be a finite field of characteristic different from $p$ such that the Sylow $p$-subgroup of $P S L_{2}\left(\mathbf{F}_{q}\right)$ has order $p$, i.e., $q^{2}-1$ is divisible by $p$ but not by $p^{2}$. Then the Sylow $p$-subgroup of $H=P S L_{2}\left(\mathbf{F}_{q^{p}}\right)$ is cyclic of order $p^{2}$. Let $\phi$ be the Frobenius automorphism of $\mathbf{F}_{q^{p}}$ of order $p$. Then it is easy to see that $\phi$ fixes a cyclic subgroup $C \leq H$ of order $p^{q}+1$ which contains one such Sylow $p$-subgroup. Thus $G=A u t(H)=P S L_{2}\left(\mathbf{F}_{q}\right) \rtimes \mathbf{Z} / p\langle\phi\rangle$ has $P=M_{3}(p)$ as a Sylow $p$-subgroup. Furthermore $N_{H}(C)$ is a dihedral group [Hu, II, 8.4 Satz] containing the permutation matrix of order two. Since this matrix is fixed by $\phi, W_{G}(P)=\mathbf{Z} / 2$ and $H^{*}(G)$ is of type (2) in Theorem 1.1 with $d=2$.

The group cohomology of a group $G$ is the cohomology of the classifying space $B G$ of $G$. The space $B G$ is stably homotopy equivalent to a wedge product of indecomposable spectra,

$$
B G \simeq X_{1} \vee X_{2} \vee \cdots \vee X_{n}
$$

A complete stable decomposition of $B G$ is a splitting into indecomposable spectra. The decomposition is unique up to stable homotopy type and ordering. If $G$ is a $p$-group, then all of these spectra are $p$-local. Otherwise, if $P$ is a Sylow $p$-subgroup of $G$, then a simple transfer argument shows the $p$-localization of $B G$ is a stable summand of $B P$,

$$
B P \simeq B G_{p} \vee Y,
$$

where $B G_{p}$ is the $p$-localization of $B G$. Hence $B G_{p}$ consists of some, but possibly not all, of the summands of $B P$. Note $H^{*}\left(B G_{p} ; \mathbf{F}_{p}\right)=H^{*}\left(B G ; \mathbf{F}_{p}\right)$.

Each indecomposable spectrum $X$ of $B P$ corresponds up to conjugacy to a primitive idempotent $e$ in the ring of stable self-maps $\{B P, B P\}$. The spectrum $X$ is the infinite mapping telescope or homotopy colimit of $e$,

$$
X \simeq e B P=T e l(B P \stackrel{e}{\rightarrow} B P)=\operatorname{hocolim}(B P \stackrel{e}{\rightarrow} B P \stackrel{e}{\rightarrow} \cdots) .
$$

For more information see either $[\mathrm{BF}]$ or [MP1]. 
For the remainder of the paper all spectra are localized at the prime $p$. If $P$ is a Swan group, then $B G \simeq B N_{G}(P) \simeq B\left(P \rtimes W_{G}(P)\right)$. Thus determining the stable homotopy type of $B G$ involves determining which summands have their cohomology left invariant by the action of the Weyl group of $G$.

$Z_{p} O u t(P) \subseteq\{B P, B P\}$ is a subring, in fact a retract. Therefore, certain indecomposable summands of $B P$ correspond to simple modules of the outer automorphism group $\operatorname{Out}(P)$. A summand corresponding to a simple $\operatorname{Out}(P)$-module is said to originate in $B P$. A summand originating in $B P$ does not occur as the summand of the classifying space of any proper subgroup of $P$.

In this paragraph we introduce some notation for Theorem 1.3 below. $L(2, k)$ originates in $B(\mathbf{Z} / p \times \mathbf{Z} / p)$ and corresponds to $S t \otimes(\text { det })^{k}$ where $S t$ is the Steinberg module for $\mathbf{F}_{p} G L_{2}\left(\mathbf{F}_{p}\right)$ and det is the determinant module. It is well known that the group ring $\mathbf{F}_{p}[\mathbf{Z} /(p-1)]$ has a complete set of orthogonal primitive idempotents $e_{0}, \ldots, e_{p-2}[\mathrm{D}]$. Lifting these idempotents to $Z_{p}[\mathbf{Z} /(p-1)]$ determines a complete stable splitting of

$$
B \mathbf{Z} / p^{n} \simeq \bigvee_{i=0}^{p-2} L(1, n, i)
$$

where $L(1, n, i)$ originates in $B \mathbf{Z} / p^{n}$. For more information on these summands see $[\mathrm{HK}]$ and $[\mathrm{D}]$.

If $P$ is a split metacyclic $p$-group, then since $W_{G}(P)$ is a $p^{\prime}$-group, we have $W_{G}(P) \leq \mathbf{Z} /(p-1)$. Thus the primitive idempotents $e_{0}, \ldots, e_{p-2}$ above determine a stable splitting of $B G$. If $P$ is non-split, then $B P$ is stably indecomposable [D, Thm. 1.3].

Among the split metacyclic groups there is one which plays a special role, the extra-special modular group $M_{3}(p)=P\left(p^{2}, p, p+1,1\right)$. It is characterized by its order and exponent which are $p^{3}$ and $p^{2}$ respectively.

Theorem 1.3. (1) If $P$ is split and $P \neq M_{3}(p)$, then

$$
\begin{gathered}
e_{0} B P=X_{0} \vee B\left(\mathbf{Z} / p^{n}\right), \quad e_{i} B P=X_{i}, \quad l=m-n, \quad 1 \leq i \leq p-2 . \\
e_{0} B P=X_{0} \vee B\left(\mathbf{Z} / p^{n}\right) \vee L(1, n, 0), \quad e_{i} B P=X_{i} \vee L(1, n, i), \quad l \neq m-n, 1 \leq i \leq p-2 .
\end{gathered}
$$

(2) If $P=M_{3}(p)$, then

$$
e_{0} B P=X_{0} \vee \bigvee_{i=0}^{p-2} L(2, i) \vee L(1,1, i), \quad e_{i} B P=X_{i}, \quad 1 \leq i \leq p-2
$$

where $X_{i}$ originates in $B P$.

(3) In both cases this yields a complete stable decomposition of BP and

$$
B G \simeq \bigvee_{i=0}^{(p-1 / d)-1} e_{i d} B P
$$


Corollary 1.4. Localized at $p$, the complete stable decomposition of $B G$ is given by one of the following:

(1) If $P$ is non-split, then $B G \simeq B P$.

(2) If $P$ is split and $P \neq M_{3}(p)$, then

$$
\begin{gathered}
B G \simeq \bigvee_{i=0}^{(p-1 / d)-1} X_{i d} \vee \bigvee_{j=0}^{p-2} L(1, n, j), \quad l=m-n . \\
B G \simeq \bigvee_{i=0}^{(p-1 / d)-1} X_{i d} \vee L(1, n, i d) \vee \bigvee_{j=0}^{p-2} L(1, n, j), \quad l \neq m-n .
\end{gathered}
$$

(3) If $P=M_{3}(p)$, then

$$
B G \simeq \bigvee_{i=0}^{(p-1 / d)-1} X_{i d} \vee \bigvee_{i=0}^{p-2} L(2, i) \vee L(1,1, i)
$$

If $P$ is split and $P \neq M_{3}(p)$, then Theorem 1.3 and Corollary 1.4 were proved by the first author [D, Thm. 1.3].

Throughout we assume $P$ is a non-abelian metacyclic $p$-group and $p$ is an odd prime. All cohomology is taken with simple coefficients in $\mathbf{F}_{p}$ and all spaces are considered stably, localized at $p$.

\section{Proofs}

The classifying space $B P$ is indecomposable if and only if $P$ is non-split $[\mathrm{D}$, Thm. 1.1]. Thus, since $B G$ is a summand in $B P$, we have $B G \simeq B P$ and $H^{*}(G) \cong H^{*}(P)$. In this case the cohomology algebras are given by [Hb, Thm. B if $m<l+q$ and Thm. E if $m=l+q]$. This completes the proof of both theorems for the non-split case.

Before turning to the proof of Theorem 1.1 we recall the notion of a Swan group [MP]. A $p$-group $P$ is called a Swan group if the cohomology of any group $G$ with $P$ as a Sylow $p$-subgroup is given by its invariants, i.e.,

$$
\text { res : } H^{*}\left(G ; \mathbf{F}_{p}\right) \stackrel{\cong}{\longrightarrow} H^{*}\left(P ; \mathbf{F}_{p}\right)^{W_{G}(P)} \text {. }
$$

The following result of Dietz and Glauberman [MP] is fundamental to our classification.

Theorem 2.1. If $P$ is a metacyclic group of odd order, then $P$ is a Swan group.

Proof of Theorem 1.1 for $P$ split. Let $\Phi(P)=\left\langle x^{p}, y^{p}\right\rangle$ be the Frattini subgroup. Then $P / \Phi(P) \cong \mathbf{Z} / p \times \mathbf{Z} / p=\langle\bar{x}, \bar{y}\rangle$. Thus quotienting by $\Phi(P)$ induces a homomorphism $\operatorname{Out}(P) \stackrel{\pi}{\longrightarrow} \operatorname{Aut}(P / \Phi(P))=G L_{2}\left(\mathbf{F}_{p}\right)$. By [D, Prop. 3.2] if $P$ is split, then $\operatorname{Out}(P) \cong O_{p} O u t(P) \rtimes \mathbf{Z} /(p-1)$; moreover,

$$
\left(\begin{array}{cc}
* & 0 \\
0 & 1
\end{array}\right) \in \operatorname{Im}(\pi)
$$


Now consider the extension

$$
1 \rightarrow N \rightarrow P \rightarrow K \rightarrow 1
$$

where $N \cong \mathbf{Z} / p^{m}=\langle x\rangle$ and $K \cong \mathbf{Z} / p^{n}=\langle\bar{y}\rangle$. The Lyndon-Hochschild-Serre spectral sequence has $E_{2}=\mathbf{F}_{p}[u, v] \otimes E[a, b]$ where $H^{*}(N)=\mathbf{F}_{p}[u] \otimes E[a], H^{*}(K)=$ $\mathbf{F}_{p}[v] \otimes E[b],|a|=|b|=1,|u|=|v|=2$. Explicitly, $a, b$ are given as canonical homomorphisms dual to $x, y$ respectively, and $u=\beta_{n}(a), v=\beta_{m}(b)$ are their respective Bocksteins.

If $l \neq m-n$, then this spectral sequence collapses at $E_{2}$ [Dh, Thm.1]. Since $P$ is a Swan group, we need only compute invariants. Let $\zeta$ be a generator of $\mathbf{Z} /(p-1) \leq$ $\operatorname{Out}(P)$ so that $\gamma=\zeta^{p-1 / d}$ generates $W_{G}(P)=\mathbf{Z} / d$. By $(1) \gamma^{*}(a)=c \cdot a$ where $c^{d}=1$ is a primitive $d$-th root of unity; $\gamma^{*}(u)=c \cdot u$ by application of the Bockstein. Similarly $\gamma^{*}$ is trivial on $v, b$. Computing we find $\gamma^{*}\left(u^{k} v^{l} a^{\epsilon} b^{\delta}\right)=u^{k} v^{l} a^{\epsilon} b^{\delta}$ iff $k+\epsilon \equiv 0 \bmod d$. Theorem 1.1 (1) follows with $u_{d}=u^{d}, a_{d}=u^{d-1} a$.

If $l=m-n$ the spectral sequence collapses at $E_{3}$ [Dh, Thm.2] and we have $E_{3}=\mathbf{F}_{p}[z, v] \otimes E\left[b, \xi_{2 i-1}, i=1, \ldots, p\right] / R$ where $z=u^{p}, \xi_{2 i-1}=a u^{i-1}$. Relations are given by

$$
\begin{gathered}
\xi_{2 i-1} \xi_{2 j-1}=0, \quad 1 \leq i, j \leq p, \\
\xi_{2 i-1} v=0, \quad 1 \leq i \leq p-1 .
\end{gathered}
$$

In this case, $\gamma^{*}(z)=c \cdot z, \gamma^{*}\left(\xi_{2 i-1}\right)=c^{i} \cdot \xi_{2 i-1}$. For $d>1$ we have invariants $z^{d}$ and $\alpha_{2 i-1}=\xi_{2 i-1} z^{d(i)}$, where $0 \leq d(i)<d$ is the residue of $-i \bmod d$. The result follows. For $d=1, H^{*}(G)=H^{*}(P)$ and the result holds setting $\alpha_{2 i-1}=\xi_{2 i-1}$ since $d(i)=0$ in this case.

Proof of Theorem 1.3 for $P=M_{3}(p)$. In this case we have [D, Thm. $1.1(3)$ ]

$$
B P \simeq \bigvee_{i=0}^{p-2} X_{i} \vee \bigvee_{k=0}^{p-2} L(2, k) \vee \bigvee_{k=0}^{p-2} L(1,1, k)
$$

Since $P$ is a Swan group, we may assume $G=N_{G}(P)$ and $C_{G}(P)<P$, i.e., $P \triangleleft G$ and $G=P \rtimes C$, where $C \leq \mathbf{Z} /(p-1)$. From (1) in the proof of Theorem 1.1 it is clear that the subgroup $\langle x\rangle \rtimes C$ is normal in $G=P \rtimes C$. Therefore, $\mathbf{Z} / p\langle y\rangle$ is a retract of $G$; hence, $B \mathbf{Z} / p$ is a summand of $B G$ for every $G$ with $M_{3}(p)$ as a Sylow $p$-subgroup. Thus

$$
B \mathbf{Z} / p=\bigvee_{k=0}^{p-2} L(1,1, k)
$$

is a summand of $e_{0} B P=B(P \rtimes \mathbf{Z} /(p-1))$.

We are reduced to showing $L(2, k)$ is a summand of $e_{0} B P$. Let $Q=\left\langle x^{p}, y\right\rangle \cong$ $\mathbf{Z} / p \times \mathbf{Z} / p$. Since $L(2, k)$ corresponds to the simple $\mathbf{F}_{p} O u t(Q)$-module $M_{k}=S t \otimes$ $(\operatorname{det})^{k}$, we can use the criterion developed in [MP1]. That is, since $Q$ is not a retract of $P$ and $C_{P}(Q)=Q$, we must show

$$
\overline{N_{G}(Q) / Q} \cdot M_{k} \neq 0
$$

where $\bar{H}=\sum h$ summed over $h \in H \leq \mathbf{F}_{p}(H)$. Since $C_{G}(Q) / Q$ is a $p^{\prime}$-group, this is equivalent to

$$
\overline{N_{G}(Q) / C_{G}(Q)} \cdot M_{k} \neq 0
$$


where $N_{G}(Q) / C_{G}(Q) \leq G L_{2}\left(\mathbf{F}_{p}\right)$. An explicit description of the Steinberg module St may be given as follows: $S t=\left\langle u^{p-1}, u^{p-2} v, \ldots, u v^{p-2}, v^{p-1}\right\rangle$ is the $\mathbf{F}_{p}$-module of polynomials in indeterminates $u, v$ of homogeneous degree $p-1$ with $G L_{2}\left(\mathbf{F}_{p}\right)$ acting on $\langle u, v\rangle$ in the standard way $[\mathrm{G}]$. Furthermore

$$
\overline{N_{G}(Q) / C_{G}(Q)}=\overline{N_{G}(Q) / P C_{G}(Q)} \cdot \overline{P / Q} .
$$

According to [D, Prop. 4.6 and Proof $]$

$$
\overline{P / Q} \cdot M_{k}=\left\langle v^{p-1}\right\rangle .
$$

Since $N_{G}(Q) / C_{G}(Q)$ is a $p^{\prime}$-group normalizing $P / Q$, we may assume it is isomorphic to a subgroup of the Borel subgroup of upper triangular matrices, i.e., the matrices of the form

$$
w=\left(\begin{array}{ll}
a & 0 \\
0 & b
\end{array}\right) .
$$

Thus $w\left(v^{p-1}\right)=b^{p-1} v^{p-1}=v^{p-1}$, and so $\overline{N_{G}(Q) / P C_{G}(Q)} \cdot v^{p-1} \neq 0$.

\section{REFERENCES}

[BF] D. Benson and M. Feshbach, Stable splittings of classifying spaces of finite groups, Topology 31 (1992), 157-176. MR 93d:55013

[Dh] T. Diethelm, The mod p cohomology rings of the non-abelian metacyclic p-groups, Arch. Math. 44 (1985), 29-38. MR 86e:20057

[D] J. Dietz, Stable splittings of classifying spaces of metacyclic p-groups, $p$ odd, J. Pure and Applied Alg. 90 (1993), 115-136. MR 95f:55014

[D1] Stable splittings of classifying spaces of metacyclic 2-groups, Math. Proc. Camb. Phil. Soc. (to appear). MR 95e:55011

[G] D. Glover, A study of certain modular representations, J. Algebra 51 (1978), 425-475. MR 57:16392

[HK] J. Harris and N. Kuhn, Stable decompositions of classifying spaces of finite Abelian pgroups, Math. Proc. Camb. Phil. Soc. 103 (1988), 427-449. MR 89d:55021

[Hb] J. Huebschmann, The mod-p cohomology rings of metacyclic groups, J. Pure and Applied Alg. 60 (1989), 53-103. MR 91a:20059

[Hu] B. Huppert, Endliche Gruppen I, Grundlehren der mathematishen Wissenschaften, vol. 134, Springer-Verlag, Berlin, Heidelberg, New York, 1967. MR 37:302

[M] J. Martino, Classifying spaces of p-groups with cyclic maximal subgroups, Topology and Representation Theory, Contemporary Mathematics, 158, Amer. Math. Soc., Providence, RI, 1994, 157-174. MR 95a:55039

[MP] J. Martino and S. Priddy, On the cohomology and homotopy of Swan groups (to appear).

[MP1] _ The complete stable splitting of the classifying space of a finite group, Topology 31 (1992), 143-156. MR 93d:55012

[MP2] Classification of BG for groups with dihedral or quaternionic Sylow 2-subgroups, J. of Pure and Applied Alg. 73 (1991), 13-21. MR 92f:55022

Department of Mathematics, University of Washington, Seattle, Washington 98195 Current address: Department of Mathematics and Computer Science, Gettysburg College, Gettysburg, Pennsylvania 17325

E-mail address: jdietz@gettysburg.edu

Department of Mathematics and Statistics, Western Michigan University, Kalamazoo, Michigan 49008

E-mail address: martino@wmich.edu

Department of Mathematics, Northwestern University, Evanston, Illinois 60208

E-mail address: s_priddy@math.nwu.edu 